\title{
Motor imagery: lessons learned in movement science might be applicable for spaceflight
}

\author{
Otmar Bock ${ }^{1 *}$, Nadja Schott ${ }^{2 \dagger}$ and Charalambos Papaxanthis ${ }^{3,4 \dagger}$ \\ ${ }^{1}$ Institute of Physiology and Anatomy, German Sport University, Köln, Germany, ${ }^{2}$ Institute of Sport and Exercise Sciences, \\ University of Stuttgart, Stuttgart, Germany, ${ }^{3}$ Université de Bourgogne, Unité de Formation et de Recherche en Sciences et \\ Techniques des Activités Physiques et Sportives, Dijon, France, ${ }^{4}$ Institut National de la Santé et de Recherche Médicale \\ (INSERM), UMR U1093, Cognition, Action, et Plasticité Sensorimotrice (CAPS), Dijon, France
}

Before participating in a space mission, astronauts undergo parabolic-flight and underwater training to facilitate their subsequent adaptation to weightlessness. Unfortunately, similar training methods can't be used to prepare re-adaptation to planetary gravity. Here, we propose a quick, simple and inexpensive approach that could be used to prepare astronauts both for the absence and for the renewed presence of gravity. This approach is based on motor imagery (MI), a process in which actions are produced in working memory without any overt output. Training protocols based on $\mathrm{Ml}$ have repeatedly been shown to modify brain circuitry and to improve motor performance in healthy young adults, healthy seniors and stroke victims, and are routinely used to optimize performance of elite athletes. We propose to use similar protocols preflight, to prepare for weightlessness, and late inflight, to prepare for landing.

Keywords: countermeasures, motor performance, mental practice, spaceflight

When humans are exposed to weightlessness during spaceflight, they typically exhibit sensorimotor deficits such as spatial disorientation, postural instability and reduced manual dexterity; the symptoms gradually subside over a period of weeks to months, but often re-appear after return to Earth (Clément and Ngo-Anh, 2013). Since these sensorimotor deficits are unpleasant, degrade task performance and increase the risk of accidents, astronauts' preflight training includes activities during parabolic flight and water immersion, where they experience weightlessness and can pre-adapt to it. Unfortunately, it is difficult to implement similar training regimes to prepare astronauts for their return to Earth, due to the limited availability of equipment and crew time. Here, we propose an approach for a pre-landing training, which requires little time and instrumentation, and which is already used with success in sports. Like astronauts, high-performance athletes face a limited availability of training equipment and a shortage of time, and an established approach to overcome these constraints is the use of motor imagery.

Motor imagery (MI) is a widely used experimental paradigm for the study of action planning and representation (Jeannerod, 1994). MI is described as an active cognitive process during which a specific action is internally reproduced in working memory, from a first-person perspective, without any overt motor output (Decety and Grèzes, 1999). It typically includes multiple sensory modalities, e.g., a person might mentally visualize her arm to move a ball, mentally feel her muscles to contract, and mentally hear the ball's "thump" against the ground 
(Weinberg, 2008). Compared to executed movements, imagined movements have similar temporal structure, accuracy and vividness (Guillot and Collet, 2005; Papaxanthis et al., 2012; Schott, 2012), activate similar brain regions in the motor and premotor cortices, the posterior parietal cortex, the basal ganglia and the cerebellum (Jeannerod, 2001; Munzert and Zentgraf, 2009), and are associated with similar autonomic responses (Decety et al., 1991; Mulder et al., 2005).

When motor imagery is scheduled repeatedly rather than just once, for the purposes of training or therapy, it is usually referred to as mental practice (MP; Driskell et al., 1994). It has been documented that MP can improve several aspects of motor performance such as muscle strength (see Yue and Cole, 1992; Ranganathan et al., 2004), movement execution and accuracy (Pascual-Leone et al., 1995; Yágüez et al., 1998; Gentili et al., 2004, 2010), and movement quality (Vogt, 1995). These changes of performance are paralleled by changes of the underlying neuronal circuitry. In a seminal study, Pascual-Leone et al. (1995) used transcranial magnetic stimulation (TMS) to investigate the cortical representation of the hand before and after a 5-day practice of a fivefinger exercise on the piano, performed physically in one group and by mental practice in another group. Both groups improved their performance in terms of accuracy and temporal consistency, and the cortical representation of long finger flexor and extensor muscles in contralateral M1 expanded similarly in both groups. Another study (Clark et al., 2014) reported that 4 weeks of wrist-hand immobilization in a control group severely reduced muscle force, impaired voluntary activation and prolonged corticospinal inhibition as assessed by TMS, while the same immobilization in an MP group that practiced 5 days per week reduced muscle force only about half as much, and completely prevented the prolongation of corticospinal inhibition. Similar effects were observed in a group of elderly patients with distal radius fracture (Schott et al., 2013a). Here, the MP group received a 6 weeks regimen consisting of $45 \mathrm{~min}$ training every weekday. A significant increase in grip strength and range of motion was observed compared to the control group. Lacourse et al. (2005) observed that 1 week of hand-movement training increases the hemodynamic response of the primary and secondary motor cortex and of the cerebellum by a comparable amount in a physical-practice and in a mental-practice group. Thus taken together, benefits of PM have been reported both at the behavioral and ant the neuronal level for healthy subjects as well as young and elderly patients.

It might seem surprising that MP enhances motor performance even though it provides no sensory feedback from body motion and the environment. The concept of internal models offers a theoretical explanation of this phenomenon. It is thought that neural mechanisms within our sensorimotor system use the currently issued motor commands to simulate the upcoming movement and its sensory consequences; the output of these so-called forward internal models (Kawato et al., 1987) is normally combined with-noisy and delayed-sensory feedback to provide accurate and precise movement estimations (Wolpert et al., 1995). Imagery of movements provides no sensory feedback, but the output of forward internal models is still available for movement estimation (Miall and Wolpert, 1996; Wolpert and Flanagan, 2001). During physical practice, movement estimations serve to refine future motor commands by generating an internal training signal that modifies plastic neural processes (Wolpert et al., 1995; Kawato, 1999; Desmurget and Grafton, 2000) and the same may hold during MP as well, only that the training signal may be less accurate and precise due to the lack of sensory feedback.

Studies on motor imagery in sports can be subdivided into three broad categories. One of them administers a single session of motor imagery along with relaxation techniques prior to a competition; this can enhance athletes' subsequent performance, probably by adjusting their arousal level and attention focus, and by reducing their anxiety (Hinshaw, 1991; Weinberg, 2008). The second category administers repeated sessions of motor imagery (i.e., MP) as well as of physical exercise; it has been shown that MP enhances progress beyond that achieved by physical practice alone, possibly by serving as a catalyst or as a genuine training stimulus (Taktek, 2004; Weinberg, 2008; Malouin et al., 2013; Vogt et al., 2013). The third category of sports-related research deals with pure mental practice; it reports that MP is efficient, albeit less efficient than pure physical practice (Hinshaw, 1991; Driskell et al., 1994; Schott et al., 2013b). This category is in use for more than 60 years (Twining, 1949); it produced training benefits on tasks ranging from muscle strength building (Wright and Smith, 2009) to gymnastic jumps (Smith et al., 2007), ball games (Wakefield and Smith, 2009; Ramsey et al., 2010) and ski slalom (Callow et al., 2006). The three categories of sports-related imagery research are not equally relevant for pre-landing countermeasures. The first category is less critical since astronauts' mental preparation for re-entry is typically well developed. The second category is unsuitable for inflight use since imagery training can't be combined with physical training under terrestrial conditions. ${ }^{1}$ The third category, however, could form a useful approach for pre-landing countermeasures.

MP has been used with success not only in sports, but also in a range of other disciplines. It has been applied to train surgical skills (Immenroth et al., 2007; Cocks et al., 2014), enhance music performance (Keller, 2012), improve balance in the elderly (Hamel and Lajoie, 2005), and support orthopedic and neurological rehabilitation (Jackson et al., 2001; Schott and Korbus, 2014). Several frameworks have been presented in the literature to define the key components of successful MP (for an overview see Guillot and Collet, 2008; Schott et al., 2013a); among them, the PETTLEP model (Holmes and Collins, 2001) emerged as the most promising one. This framework is elaborated by the Functional Equivalence Model, which states

\footnotetext{
${ }^{1}$ Although payload such as treadmills equipped with bungee cords is available aboard the ISS, such hardware simulates some but not other impacts of terrestrial gravity. This hardware is valuable for the training of strength and endurance, but it would be inadequate for the training of sensorimotor coordination where it even might produce negative transfer.
} 
that motor imagery should resemble the actual task in seven critical aspects, summarized by the acronym PETTLEP (Holmes and Collins, 2001). This acronym stands for Physical (body should assume the same physical postures as in the actual task, rather than being relaxed as in many earlier studies), Environment (imagery should include the same architecture and furnishing as the actual task), Task (same body and eye movements, muscle tensions, heart rate, dry mouth etc. as in actual task), Timing (not in slow motion as in some earlier studies), Learning (imagery should be modified as practice progresses), Emotion (same excitement and arousal as with actual task, but avoid negative feelings) and Perspective (the 1st person perspective is more efficient in most tasks, but there are exceptions).

It became standard practice within the last decade to guide subjects' motor imagery by scripts. This approach has been formalized in detail by Eberspächer and colleagues (Immenroth et al., 2007). First, experimenter and trainees observe and discuss the desired motor activity and its sensory, autonomic and emotional concomitants. The outcome is then fixed in written form to enhance precision. The activity is subsequently broken down into key components or "nodal points", and each nodal point obtains a verbal label such that calling it up is facilitated. In a final step, the key characteristics are marked symbolically, i.e., they are renamed as individual short formulae (e.g., up-centered or pull-release for dorsal flexion). The aim is the compression of the information (cognitive chunking) of body postures and movements. The image should thus be approximated to the dynamics and temporal duration of the real movement. In each session, the trainee reads or hears the script and then engages in the pertinent imagery. Due to findings of Yao et al. (2013), that mental imagery is most effective with a kinesthetic focus, the training process should aim at that modality.

A series of experiments by Smith and colleagues (Smith et al., 2007) provides good examples for successful motor imagery training based on PETTLEP and scripting. The experimenter formulated with each trainee an individualized script which included descriptions of visual and kinesthetic inputs, motor outcomes, smells and emotions; these scripts were modified as practice progressed, to satisfy the Learning principle of PETTLEP. Imagery sessions were scheduled in the trainees' normal sports facility, with participants wearing their normal sports clothes. PETLEP training was not as effective as physical training, but was more effective than standard imagery in seated and relaxed subjects.

Given its success on Earth, MP based on PETTLEP and scripting could also be useful for pre-adapting astronauts to the terrestrial environment while they are still in orbit. We suggest a training regime where individualized scripts are established and refined before launch, and are used for MP during the final days before landing.

The first phase would take place on Earth, in the astronauts' familiar environment. They would wear their regular outfit and perform their typical daily activities such as standing up, walking with and without a load, sitting down, manually retrieving, handling and replacing of objects, etc. Astronauts would then engage in mental practice of these activities, using the scripts, to provide the individually relevant functional, sensory and emotional aspects of the reference movements, their dynamics and their timing. In a next step, astronauts would elaborate the key characteristics of the activities in form of scripts. To this end, they assume and maintain the starting posture of the respective physical movement, execute the activities with eyes open, with eyes closed (to enhance kinesthetic perception, see Yao et al., 2013) and mentally, and then report their sensations, observations and emotions to the instructor. Once the scripts are formulated in several iterative steps, the key movement characteristics are marked symbolically, e.g., the sequence "stand up-walk-turn-walk-sit down" could be labeled "TUG", as in the timed-up-and-go test. To assess training progress, astronauts would signal the onset and completion of each imagined sub-movement to the observer by slightly lifting their hand; the systematic and variable errors between the timing of imagined and physical movements could then serve as markers of progress.

The second phase would start several days before landing, and consist of multiple sessions in which astronauts listen to the short-formed scripts and then mentally imagine the pertinent sensations, observations and emotions. They would not engage in those activities physically, since in the absence of gravity, this would defeat the purposes of training. Again, astronauts would use hand signals to allow judgments of their progress.

We expect that in analogy to MP in sports, MP before landing will improve astronauts' sensorimotor performance upon return to Earth, and will thus serve as an effective countermeasure. For example, practice of the above "TUG" script could enhance astronauts' ability for standing up and walking without assistance after landing, and the benefit could generalize to other postural tasks as well.

Mental practice could be applied to prepare astronauts not only for the sudden onset of gravity upon landing, but also to the sudden absence of gravity after launch. Presently, this preparation is limited to parabolic flights-which offer only brief periods of weightlessness that alternate with periods of hypergravity, and to water immersion-which introduces confounding factors such as viscosity, slight nitrogen narcosis (Dalecki et al., 2012) and persistence of normal vestibular inputs. Mental practice could offer an expedient alternative or supplement to those established methods. Likewise, mental practice could be applied to prepare astronauts for the landing on and the launching from celestial bodies other than the Earth. Finally, MP could be used as countermeasure for decrements not only of motor skill, but also of muscular force: particularly MP with kinesthetic imagery was found to counteract the loss of muscle strength following immobilization (Meugnot et al., 2015) without inducing muscular fatigue (Rozand et al., 2014); the effects of MP on muscular force are not much smaller than those of physical practice (Reiser et al., 2011). 


\section{References}

Callow, N., Roberts, R., and Fawkes, J. Z. (2006). Effects of dynamic and static imagery on vividness of imagery, skiing performance and confidence. J. Imagery Res. Sport Phys. Act. 1, 1-82. doi: 10.2202/1932-0191.1001

Clark, B. C., Mahato, N. K., Nakazawa, M., Law, T. D., and Thomas, J. S. (2014). The power of the mind: the cortex as a critical determinant of muscle strength/weakness. J. Neurophysiol. 112, 3219-3226. doi: 10.1152/jn.00386. 2014

Clément, G., and Ngo-Anh, J. T. (2013). Space physiology II: adaptation of the central nervous system to space flight-past, current and future studies. Eur. J. Appl. Physiol. 113, 1655-1672. doi: 10.1007/s00421-012-2509-3

Cocks, M., Moulton, C., Luu, S., and Cil, T. (2014). What surgeons can learn from athletes: mental practice in sports and surgery. J. Surg. Educ. 71, 262-269. doi: 10.1016/j.jsurg.2013.07.002

Dalecki, M., Bock, O., and Schulze, B. (2012). Cognitive impairment during $5 \mathrm{~m}$ water immersion. J. Appl. Physiol. (1985) 113, 1075-1081. doi: 10. 1152/japplphysiol.00825.2012

Decety, J., and Grèzes, J. (1999). Neural mechanisms subserving the perception of human actions. Trends Cogn. Sci. 3, 172-178. doi: 10.1016/s13646613(99)01312-1

Decety, J., Jeannerod, M., Germain, M., and Pastene, J. (1991). Vegetative response during imagined movement is proportional to mental effort. Behav. Brain Res. 42, 1-5. doi: 10.1016/s0166-4328(05)80033-6

Desmurget, M., and Grafton, S. (2000). Forward modeling allows feedback control for fast reaching movements. Trends Cogn. Sci. 4, 423-431. doi: 10.1016/s13646613(00)01537-0

Driskell, J., Copper, C., and Moran, A. (1994). Does mental practice enhance performance? J. Appl. Psychol. 79, 481-492. doi: 10.1037/0021-9010.79.4.481

Gentili, R., Cahouet, V., Ballay, Y., and Papaxanthis, C. (2004). Inertial properties of the arm are accurately predicted during motor imagery. Behav. Brain Res. 155, 231-239. doi: 10.1016/j.bbr.2004.04.027

Gentili, R., Han, C. E., Schweighofer, N., and Papaxanthis, C. (2010). Motor learning without doing: trial-by-trial improvement in motor performance during mental training. J. Neurophysiol. 104, 774-783. doi: 10.1152/jn.00257. 2010

Guillot, A., and Collet, C. (2005). Duration of mentally simulated movement: a review. J. Mot. Behav. 37, 10-20. doi: 10.3200/jmbr.37.1.10-20

Guillot, A., and Collet, C. (2008). Construction of the motor imagery integrative model in sport: a review and theoretical investigation of motor imagery use. Int. Rev. Sport Exerc. Psychol. 1, 31-44. doi: 10.1080/17509840701823139

Hamel, M. F., and Lajoie, Y. (2005). Mental imagery. Effects on static balance and attentional demands of the elderly. Aging Clin. Exp. Res. 17, 223-228. doi: 10. 1007/bf03324601

Hinshaw, K. E. (1991). The effects of mental practice on motor skill performance: critical evaluation and meta-analysis. Imagin. Cogn. Pers. 11, 3-35. doi: 10. 2190/x9ba-kj68-07an-qmj8

Holmes, P. S., and Collins, D. J. (2001). The PETTLEP approach to motor imagery: a functional equivalence model for sport psychologists. J. Appl. Sport Psychol. 13, 60-83. doi: 10.1080/104132001753155958

Immenroth, M., Bürger, T., Brenner, J., Nagelschmidt, M., Eberspächer, H., and Troidl, H. (2007). Mental training in surgical education: a randomized controlled trial. Ann. Surg. 245, 385-391. doi: 10.1097/01.sla.0000251575. 95171.b3

Jackson, P. L., Lafleur, M. F., Malouin, F., Richards, C., and Doyon, J. (2001). Potential role of mental practice using motor imagery in neurologic rehabilitation. Arch. Phys. Med. Rehab. 82, 1133-1141. doi: 10.1053/apmr.2001. 24286

Jeannerod, M. (1994). The representing brain: neural correlates of motor intention and imagery. Behav. Brain Sci. 17, 187-245. doi: 10.1017/s0140525x00 034026

Jeannerod, M. (2001). Neural simulation of action: a unifying mechanism for motor cognition. Neuroimage 14, S103-S109. doi: 10.1006/nimg.2001.0832

Kawato, M. (1999). Internal models for motor control and trajectory planning. Curr. Opin. Neurobiol. 9, 718-727. doi: 10.1016/s0959-4388(99)00028-8

Kawato, M., Furukawa, K., and Suzuki, R. (1987). A hierarchical neural-network model for control and learning of voluntary movement. Biol. Cybern. 57, 169-185. doi: 10.1007/bf00364149
Keller, P. E. (2012). Mental imagery in music performance: underlying mechanisms and potential benefits. Ann. N Y Acad. Sci. 1252, 206-213. doi: 10. 1111/j.1749-6632.2011.06439.x

Lacourse, M. G., Orr, E. L. R., Cramer, S. C., and Cohen, M. J. (2005). Brain activation during execution and motor imagery of novel and skilled sequential hand movements. Neuroimage 27, 505-519. doi: 10.1016/j.neuroimage.2005. 04.025

Malouin, F., Jackson, P. L., and Richards, C. L. (2013). Towards the integration of mental practice in rehabilitation programs. A critical review. Front. Hum. Neurosci. 7:576. doi: 10.3389/fnhum.2013.00576

Meugnot, A., Agbangla, N. F., Almecija, Y., and Toussaint, L. (2015). Motor imagery practice may compensate for the slowdown of sensorimotor processes induced by short-term upper-limb immobilization. Psychol. Res. 79, 489-499. doi: 10.1007/s00426-014-0577-1

Miall, R. C., and Wolpert, D. M. (1996). Forward models for physiological motor control. Neural Netw. 9, 1265-1279. doi: 10.1016/s0893-6080(96)00035-4

Mulder, T., de Vries, S., and Zijlstra, S. (2005). Observation, imagination and execution of an effortful movement: more evidence for a central explanation of motor imagery. Exp. Brain Res. 163, 344-351. doi: 10.1007/s00221-004-2179-4

Munzert, J., and Zentgraf, K. (2009). Motor imagery and its implications for understanding the motor system. Prog. Brain Res. 174, 219-229. doi: 10. 1016/s0079-6123(09)01318-1

Papaxanthis, C., Paizis, C., White, O., Pozzo, T., and Stucchi, N. (2012). The relation between geometry and time in mental actions. PLoS One 7:e51191. doi: 10.1371/journal.pone.0051191

Pascual-Leone, A., Nguyet, D., Cohen, L. G., Brasil-Neto, J. P., Cammarota, A., and Hallett, M. (1995). Modulation of muscle responses evoked by transcranial magnetic stimulation during the acquisition of new fine motor skills. J. Neurophysiol. 74, 1037-1045.

Ramsey, R., Gumming, J., Edwards, M. G., Williams, S., and Brunning, C. (2010). Examining the emotion aspect of PETTLEP-based imagery with penalty taking in soccer. J. Sport Behav. 33, 295-314.

Ranganathan, V. K., Siemionow, V., Liu, J. Z., Sahgal, V., and Yue, G. H. (2004) From mental power to muscle power-gaining strength by using the mind. Neuropsychologia 42,944-956. doi: 10.1016/j.neuropsychologia.2003.11.018

Reiser, M., Büsch, D., and Munzert, J. (2011). Strength gains by motor imagery with different ratios of physical to mental practice. Front. Psychol. 2:194. doi: 10. 3389/fpsyg.2011.00194

Rozand, V., Lebon, F., Papaxanthis, C., and Lepers, R. (2014). Does a mental training session induce neuromuscular fatigue? Med. Sci. Sports Exerc. 46, 1981-1989. doi: 10.1249/MSS.0000000000000327

Schott, N. (2012). Predictors of adult age differences in motor imagery. Exp. Aging Res. 38, 1-26. doi: 10.1080/0361073X.2012.726045

Schott, N., Frenkel, M.-O., Korbus, H., and Francis, K. L. (2013a). Mental practice in orthopedic rehabilitation: where, what and how? A case report. Sci. Motricité 82, 93-103. doi: 10.1051/sm/2013099

Schott, N., Hohmann, T., and Schneider, F. (2013b). Does motor imagery ability influence the learning rate of a complex reach-to-grasp movement? J. Sport Exerc. Psychol. 35, S50.

Schott, N., and Korbus, H. (2014). Preventing functional loss during immobilization after osteoporotic wrist fractures in elderly patients: a randomized clinical trial. BMC Musculoskelet. Disord. 15:287. doi: 10. 1186/1471-2474-15-287

Smith, D., Wright, C., Allsopp, A., and Westhead, H. (2007). It's all in the mind: PETTLEP-based imagery and sports performance. J. Appl. Sport Psychol. 19, 80-92. doi: 10.1080/10413200600944132

Taktek, K. (2004). The effects of mental imagery on the acquisition of motor skills and performance: a literature review with theoretical implications. J. Ment. Imagery 28, 79-114.

Twining, W. E. (1949). Mental practice and physical practice in learning a motor skill. Res. Q. 20, 432-435.

Vogt, S. (1995). On relations between perceiving, imagining and performing in the learning of cyclical movement sequences. Br. J. Psychol. 86, 191-216. doi: 10. 1111/j.2044-8295.1995.tb02556.x

Vogt, S., Di Rienzo, F., Collet, C., Collins, A., and Guillot, A. (2013). Multiple roles of motor imagery during action observation. Front. Hum. Neurosci. 7:807. doi: 10.3389/fnhum.2013.00807 
Wakefield, C. J., and Smith, D. (2009). Impact of differing frequencies of PETTLEP imagery on netball shooting performance. J. Imagery Res. Sport Phys. Act. 4:7. doi: 10.2202/1932-0191.1043

Weinberg, R. (2008). Does imagery work? Effects on performance and mental skills. J. Imagery Res. Sport Phys. Act. 3:1. doi: 10.2202/1932-0191. 1025

Wolpert, D. M., and Flanagan, J. R. (2001). Motor prediction. Curr. Biol. 11, R729-R732. doi: 10.1016/S0960-9822(01)00432-8

Wolpert, D., Ghahramani, Z., and Jordan, M. (1995). An internal model for sensorimotor integration. Science 269, 1880-1882. doi: 10.1126/science. 7569931

Wright, C. J., and Smith, D. (2009). The effect of PETTLEP imagery on strength performance. Int. J. Sport Exerc. Psychol. 7, 18-31. doi: 10.1080/1612197x.2009. 9671890

Yágüez, L., Nagel, D., Hoffman, H., Canavan, A. G., Wist, E., and Hömberg, V. (1998). A mental route to motor learning: improving trajectorial kinematics through imagery training. Behav. Brain Res. 90, 95-106. doi: 10.1016/s01664328(97)00087-9
Yao, W. X., Ranganathan, V. K., Allexandre, D., Siemionow, V., and Yue, G. H. (2013). Kinesthetic imagery training of forceful muscle contractions increases brain signal and muscle strength. Front. Hum. Neurosci. 7:561. doi: 10. 3389/fnhum.2013.00561

Yue, G., and Cole, K. J. (1992). Strength increases from the motor program: comparison of training with maximal voluntary and imagined muscle contractions. J. Neurophysiol. 67, 1114-1123.

Conflict of Interest Statement: The authors declare that the research was conducted in the absence of any commercial or financial relationships that could be construed as a potential conflict of interest.

Copyright (c) 2015 Bock, Schott and Papaxanthis. This is an open-access article distributed under the terms of the Creative Commons Attribution License (CC BY). The use, distribution and reproduction in other forums is permitted, provided the original author(s) or licensor are credited and that the original publication in this journal is cited, in accordance with accepted academic practice. No use, distribution or reproduction is permitted which does not comply with these terms. 